\title{
Validation of Lower-Bound Estimates for Compression-Loaded Cylindrical Shells
}

\author{
Waddy T. Haynie* and Mark W. Hilburger ${ }^{\dagger}$ \\ NASA Langley Research Center, Hampton, VA, 23681, USA \\ Massimiliano Bogge $e^{\ddagger}$ and Marco Maspoli ${ }^{\ddagger}$ \\ Politecnico di Torino, Italy, 10129 \\ Benedikt Kriegesmann ${ }^{\S}$ \\ Leibniz Universität Hannover, Germany, 30167
}

\begin{abstract}
The traditional approach used in the design of stability critical thin-walled circular cylinders, is to reduce unconservative buckling load predictions with an empirical knockdown factor. An alternative analysis-based approach to determine a lower bound buckling load for cylinders under axial compression is to use a lateral perturbation load to create an initial imperfection and determine the buckling load while that load is applied. This paper describes a preliminary effort to develop a test capability to verify this approach. Results from tests of three aluminum alloy cylinders are described and compared to finite element predictions.
\end{abstract}

\section{Introduction}

I IGHT-WEIGHT thin-walled, circular cylindrical shells used in aerospace applications are inherently stability Lcritical structures. Considering circular cylinders under an axial compressive load, linear buckling analyses predict unconservative buckling loads when compared to corresponding experimental results. Having an unconservative prediction and uncertainty in the buckling load of an as-built structure, presents a significant challenge to the designer of such structures. In the design of thin-walled circular cylinders, the traditional approach is to reduce the buckling load predicted from a deterministic, linear bifurcation analysis with an empirical knockdown factor (e.g., Ref 1). The knockdown factor has been shown to result in overly conservative designs, but it can potentially result in unconservative designs if the data with which it was determined are not representative of the design of interest.

Initial geometric imperfections in the cylinder walls are recognized as a major contributor to the discrepancy between analytically predicted buckling loads for cylinder and the corresponding experimentally obtained buckling loads (e.g., Refs 2-6). The sensitivity of the response of a cylinder to various forms of imperfections is not included in traditional sources of design knockdown factors. Krasovsky et al. ${ }^{7}$ and Hühne et al. ${ }^{8}$ have recently performed numerical and experimental studies on cylinders where a lateral perturbation load is used to create a dimple-shaped imperfection in the cylinder wall. This dimple is similar to the type of dimple that forms at the onset of buckling in a test of a cylinder under axial compression, so therefore with the artificially induced imperfection there is a link to the buckling behavior of an un-perturbed cylinder. A lower bound for the buckling load can also be determined using the lateral perturbation load. For smaller magnitudes of the lateral perturbation load, the buckling load is highly sensitive to changes in the magnitude of the perturbation load, with the buckling load decreasing with an increasing perturbation load. However, above a threshold value of the perturbation load the buckling load is not sensitive to changes in the magnitude of the perturbation load, and the buckling load changes very little with an increase in the perturbation load. The buckling load at the threshold value is an approximation of a lower bound buckling load for the cylinder.

\footnotetext{
* Research Aerospace Engineer, Structural Mechanics and Concepts Branch, Mail Stop 190. Member, AIAA

${ }^{\dagger}$ Research Aerospace Engineer, Structural Mechanics and Concepts Branch, Mail Stop 190. Senior Member, AIAA.

${ }^{\ddagger}$ Visiting NIA Research Assistant, Corso Duca degli Abruzzi, 24 - 10129.

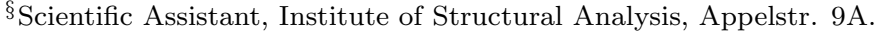


This paper presents a preliminary effort to develop a test capability to verify the approach of predicting the lower bound buckling load using the lateral perturbation load for cylinders under axial compression. The experimental set-up will be discussed. Detailed finite element analyses have also been used for pretest predictions, and have been used after testing to correlate the numerical response to the experimental response of the cylinders.

\section{Test Article Description}

Three circular cylinders were tested under an axial compressive load and with a lateral perturbation load applied in the radial direction at the mid-length of the cylinder. The cylinders were constructed from 0.040-inch thick 2024 aluminum alloy sheets that were rolled into a circular shape. To hold the cylinder together, two straps of the same material were bonded to the inside and outside of the seam created by the edges of the sheet when it was bent into the circular shape. A row of rivets was then added on each side of the seam. To simulate a clamped boundary condition, one inch of each end of the cylinder was potted in an epoxy grout. After being potted the ends of the cylinder were machined flat and parallel to ensure uniform loading. The geometry and coordinate system of the cylinders are described in figure 1 , where $R, L$, and $H$ denote the radius, length, and wall thickness, respectively, of the cylinder. The axial coordinate, $x$, and the circumferential coordinate, $s$, describe the cylinder surface. The $z$ coordinate describes the direction normal to the surface. Axially, the origin is at one end of the cylinder, and circumferentially it is at the center of the riveted strap. Not shown in figure 1 are the displacements $u, v$, and $w$, corresponding to the directions $x$, $s$, and $z$, respectively. The geometry and material properties of the cylinder are given in Table 1 . Note that the length, $L=31 \mathrm{in}$., given in Table 1 is the length of the test section; because each end of the cylinder is potted, the overall length of the cylinder is $33 \mathrm{in}$.

\section{Experimental Set-Up}

The experimental set-up is shown in figure 2. The cylinders were tested in a 600-kip load frame, and loaded under a constant rate of displacement. Three linear variable displacement transducers (LVDT's) were used to measure the displacement and rotation of the loading platens during the test. A second coordinate system was defined to describe the platen rotation. This coordinate system is shown along with the positive directions of the platen rotations, $\alpha_{Y}$ and $\alpha_{Z}$, in figure 3. The X-axis in the platen coordinate system is parallel with the $x$-axis in the surface coordinate system for the cylinder.

Three three-dimensional digital image correlation (DIC) systems were used to capture the full-field displacement response of the outer surface of the cylinder during loading. Strain gages were installed on the test articles and used for localized strain measurements. Note the cylinder shown in figure 2 was used as a checkout specimen, and in that case, the cylinder was bolted to load rings instead of having the ends potted. No results are presented in this paper for this specimen. All results presented in this paper are for cylinders with potted ends as described above.

The lateral perturbation apparatus is also shown in figure 2, with several parts of the apparatus labeled. The frame of the apparatus was constructed from extruded aluminum profiles. The apparatus used a twopulley system to push a rod into the side of a cylinder to create the lateral load. A rope, fixed on one end, was wrapped around the pulley attached to the end of the rod, then wrapped around the fixed pulley. The other end of the rope was attached to a hanger. Weights were added to the hanger to generate the lateral

perturbation load. Two linear bearings where used to constrain the rod to translate along a fixed axis, and ensure that the lateral load did not change direction during the test. The apparatus was placed so the lateral load was applied at a circumferential location of 180 degrees (diametrically opposite from the riveted strap), and at the mid-length in the axial direction. A close-up view of the end of the rod that contacts the cylinder is shown in the inset image in figure 2. A 150-lb load cell, shown in the inset image, was attached to the end of the rod, and a 0.5-in. half-spherical tip was attached to the other end of the load cell. This load cell was used to measure the magnitude of the applied lateral load. A 3-in. LVDT was used to measure the displacement of the rod during a test. 


\section{Finite Element Models and Analyses}

Finite element analyses were conducted to determine which cases to consider during testing. Analyses were run using Abaqus Standard ${ }^{9}$ for quasi-static analyses and Abaqus Explicit ${ }^{10}$ for transient analyses. The models were created using user-written codes for mesh generation. A representative finite element mesh is shown in figure 4. The mesh was comprised of S4R shell elements with an element length of approximately 0.28 in. in both the axial and circumferential directions. The elements in the area of the strap, denoted by the darker-shaded elements in figure 4, were defined with a thickness three times the thickness of the rest of the cylinder. As shown in figure 4, the origin of the coordinate system was at the center of the strap. At each end of the cylinder, a node, herein referred to as the central node, was defined at the center of the circular cross section (this node is denoted by the white $\times$ at $x=0$ in figure 4). Rigid links, not shown in figure 4 , were defined to connect each of the nodes at the end of the cylinder to the central node. The rigid links constrain the displacements $u, v$, and $w$ of the nodes at the end of the cylinder from moving with respect to the central node. The rotational degrees of freedom $\phi_{x}, \phi_{s}$, and $\phi_{z}$ of the nodes were also constrained from rotating with respect to the ends of the rigid links. Using these constraints one end of the cylinder was completely clamped by fixing the central node at $x=L$. At the other end, $x=0$, a clamped boundary condition was enforced, but the end of the cylinder was loaded by applying an axial displacement, $\Delta$, to the central node.

Two types of analyses were used to predict the behavior of the cylinders. For the perfect cylinder, the case with no lateral load, a geometrically nonlinear transient analysis was run to be able to capture the buckling and initial postbuckling response of the cylinder. For the cases with a lateral load applied, geometrically nonlinear static analyses with automatic stabilization were used to predict the response. Automatic stabilization is a capability within Abaqus Standard that allows for a stable path to found when an unstable solution step is encountered. Each case with a lateral load consisted of two steps. In the first, only the lateral load was applied; and then in the second, with the lateral load still being applied, an axial end displacement was applied. The analysis was continued until a global instability was encountered.

\section{Test and Analysis Results and Discussion}

In this section, finite element predictions and experimental results will be presented for cylinders with the geometry described above. Initial finite element predictions are presented in order to describe how the test cases were determined. Then, results from the tests of three different cylinders are presented, and the behavior of the lateral perturbation apparatus during testing is described. Lastly, representative results from modified finite element analyses used to correlate with the experimental results are presented.

\section{A. Initial finite element predictions}

Predictions from finite element analyses for several magnitudes of lateral loads were used to determine the cases that would be considered for testing. The case with no lateral load applied to the cylinder was also considered, and is referred to herein as the perfect cylinder. Finite element predictions of the loadend displacement response are shown in figure 5. The curves for each case end just prior to global buckling. Predictions of the radial displacement at the point at which the lateral load was applied are shown in figure 6, and these responses also end just prior to global buckling. The predicted relationship of the critical loads to the lateral load is shown in figure 7. Critical loads for local and global buckling are shown in the figure.

The predicted behavior can be separated into three zones as designated in figure 7 (similar behavior to these zones was described by Hühne et al. ${ }^{8}$ and Haynie and Hilburger ${ }^{11}$ ). In zone 1, represented by lateral loads of $Q=6.74$ and $11.24 \mathrm{lb}$, the lateral load had no effect on the prebuckling stiffness of the cylinder, as shown in figure 5 where the slope of the load-end shortening predictions are the same as the slope of the perfect cylinder. The radial displacements at the point of application of the lateral load were small for the two cases in zone 1 , with the largest magnitude approximately one wall thickness $(0.04 \mathrm{in}$.). In zone 2 , for the range of lateral loads $14.70 \mathrm{lb}<Q<24.7 \mathrm{lb}$, a local buckling event occurred with only the area near the point where the lateral load was applied undergoing any significant deflection. In figures 5 and 6 , this local buckling is identified by the parts of the curves with nearly vertical slopes. The change in shape from before and after this local buckling event is depicted in figures 8 and 9. In these figures the shapes are depicted using color contours of the radial displacement plotted on the rolled-out surface of the cylinder. As shown in figure 5, prior to local buckling the slope of the load-end displacement response was the same as the perfect 
cylinder. After local buckling the slope has decreased slightly. For all of the cases of lateral loads in zone 2, the global buckling load was greater than the local buckling load. In zone 3, for lateral loads of $Q>24.7 \mathrm{lb}$, benign local buckling occurred, resulting in a change in slope of the load-end displacement curve (figure 5), and a significant increase in the radial displacement at the point of application of the lateral load (figure 6). In all three zones, the predicted global postbuckling shape was approximately the same. The postbuckling shape is shown in figure 10 .

Using the methodology described by Hühne et al., ${ }^{8}$ the lower bound buckling load can be established from zone 2 or 3 , where the global buckling load is not sensitive to changes in the magnitude of the lateral load. For this cylinder geometry, and using the data shown in figure 7, the predicted lower bound buckling load would be 40 kips.

\section{B. Test results}

Based on the finite element predictions discussed above, two lateral load magnitudes, $Q=14.7$ and 24.7 $\mathrm{lb}$, were chosen for experimental validation. These loads were near the bounds of zone 2 in figure 7 . Three cylinders designated as TA01, TA02, and TA06 were loaded under an axial compressive load until buckling occurred. A lateral load of $Q=14.7 \mathrm{lb}$ was applied to TA01 and TA06, and a lateral load of $Q=24.7 \mathrm{lb}$ was applied to TA02. For TA02, one case was loaded only until a local buckling event occurred, and this case is designated as TA02-local. The second case with TA02 was loaded until global buckling occurred, and this case is designated as TA02-global. For all test articles, when global buckling occurred, plastic deformation in the cylinder prevented the cylinder from being tested again.

The axial load-end displacement measurements for all four test cases are shown in figure 11. The end displacements were modified so a linear fit of the initial prebuckling part of the response would pass through the origin of the plot. This modification accounts for the nonlinearity at the beginning of the response due to settling of the test specimen in the test frame. The responses end at the point when global buckling occurred. As shown in the finite element predictions (figure 5), when local buckling would occur in both cases for TA02, there should be a change in the slope of the axial load-end displacement response, and the load would continue to increase with increasing end displacement. As shown in figure11, TA02 did exhibit a change in slope after the local buckling event. On the other hand, after the initial nonlinearity, the two test articles TA01 and TA06 did not exhibit local buckling, and had a constant slope until buckling occurred.

Behavior similar to the finite element predictions was also seen in the radial displacement at the point of application of the lateral load. The radial displacement for this point is shown in figure 12 for the four test cases. For the two cases that did not exhibit local buckling, TA01 and TA06, the radial displacement prior to buckling was less than a wall thickness. When local buckling did occur, as with both cases of TA02, the radial displacement increased an order of magnitude to approximately $0.4 \mathrm{in}$. Then, as the end displacement was increased, the magnitude of the radial displacement continued to increase until global buckling occurred.

Similar behavior between the full-field radial displacement predictions and measurements was also observed. Using the results from the three DIC systems for TA02-global, color contours of the measured full-field radial displacement superimposed on the cylinder surface just prior to local buckling, after local buckling, and after global buckling are shown in figures 13, 14, and 15, respectively. The global buckling shape shown in figure 15 is representative of the shapes observed in TA01 and TA06. The circumferential locations are denoted by the white lines in the figures and represent circumferential locations on the undeformed cylinder and do not necessarily conform to the deformed shape of the cylinder. In all three instances, the shapes observed during the test were similar to the predicted shapes shown in figures 8, 9, and 10 .

Despite the qualitative agreement in the axial load and radial displacement responses between the finite element predictions and experimental measurements, the predictions of the local and global buckling loads were significantly greater than the measured buckling loads. These loads are summarized in Table 2. For all buckling loads, local and global, the prediction was at least $5.5 \%$ greater than the measured value, and for most cases the predicted load was more that $10 \%$ greater than the measured buckling load.

To investigate the discrepancy in the predicted and measured buckling loads, another type of imperfection was considered. Specifically, a load imperfection resulting from the rotation of the platen during testing was examined. Platen rotations $\alpha_{Y}$ and $\alpha_{Z}$ for the TA02-global case are shown in figure 16. The largest change in rotation occurred after local buckling which is indicated by the almost infinite slope of the $\alpha_{Y}$ response near an end displacement value of 0.03 in. After local buckling, this displacement continued to increase approximately linearly until global buckling occurred. The change in the rotation angle from the initiation of local buckling to the initiation of global buckling was approximately 0.024 degrees. When modifying the 
finite element analysis, a platen rotation angle of 0.02 degrees was used. The platen rotation was added to the analysis before the lateral load was applied, and was applied by applying a rotation to the central node (figure 4). The resulting axial load-end displacement response for the analysis with the platen rotation included is shown in figure 17, along with the original response curve for the analysis with no rotation, and the measured response curve for TA02-global. When the platen rotation was included in the analysis, the global buckling load was 37.5 kips, which was $1 \%$ less than the measured global buckling load for TA02global. The improved agreement between the predicted and measured buckling loads indicates that for this geometry, which did not have axisymmetric axial stiffness due to the greater thickness of the cylinder wall at the riveted strap, that when predicting a lower bound buckling load other imperfections must be considered in addition to the imperfection created by the applied lateral load.

\section{Concluding Remarks}

A first series of tests in an effort to develop the capability to verify the approach of predicting a lower bound buckling load using a lateral perturbation load for cylinders under axial compression was presented. An apparatus to apply a lateral load to the cylinders was developed, and three aluminum alloy cylinders were tested for two magnitudes of lateral loads. Good qualitative agreement between finite element predictions and experimental results were obtained; however, the finite element predictions of buckling loads were unconservative. Follow-on finite element analyses that included the rotation of the loading platen measured during testing showed that for this particular geometry that form of nonuniform loading was significant when trying to predict a lower bound buckling load using the lateral perturbation approach.

\section{Acknowledgments}

This work was conducted as part of the NASA Engineering and Safety Center (NESC) Shell Buckling Knockdown Factor Project, NESC Assessment \#:07-010-E.

\section{References} 1965.

${ }^{1}$ Anon., "Buckling of Thin-Walled Circular Cylinders," NASA Space Vehicle Design Criteria, NASA SP-8007, September

${ }^{2}$ Koiter, W. T., On the Stability of Elastic Equilibrium, (in Dutch), H. J. Paris, Amsterdam, Holland, 1945.

${ }^{3}$ von Kármán, T. and Tsien, H.-S., "The Buckling of Thin Cylindrical Shells Under Axial Compression," Journal of the Aeronautical Sciences, Vol. 8, No. 8, 1941, pp. 301-312.

${ }^{4}$ Budiansky, B. and Hutcinson, J., "Dynamic Buckling of Imperfection Sensitive Structures," Proceedings of the 11th IUTAM Congress, edited by H. Gortler, Springer-Verlag, Berlin, 636-651 1964.

${ }^{5}$ Árbocz, J. and Babcock, C. D., "The Effect of General Imperfections on the Buckling of Cylindrical Shells," Journal of Applied Mechanics, Vol. 36, No. 1, 1969, pp. 28-38.

${ }^{6}$ Sechler, E. E., "The Historical Development of Shell Research and Design," Thin-Shell Structures, Theory, Experiments, and Design, edited by Y. C. Fung and E. E. Sechler, Prentice-Hall, Englewood Cliffs, NJ, 1974, pp. 3-25.

${ }^{7}$ Krasivsky, V., Marchenko, V., and Schmidt, R., "Deformation and buckling of Axially Compressed Cylindrical Shells with Local Loads in Numerical Simulation and Experiments," Thin-Walled Structures, Vol. 49, No. 5, May 2011, pp. 576-580.

${ }^{8}$ Hühne, C., Rolfes, R., Breitbach, E., and Teßmer, J., "Robust Design of Composite Cylindrical Shells under Axial Compression - Simulation and Validation," Thin-Walled Structures, Vol. 46, 2008, pp. 947-962.

${ }^{9}$ Software Package, Abaqus/Standard, SIMULIA, Providence, RI, ver. 2011.11.1 ed., 2011.

${ }^{10}$ Software Package, Abaqus/Explicit, SIMULIA, Providence, RI, ver. 2011.11.1 ed., 2011.

${ }^{11}$ Haynie, W. T. and Hilburger, M. W., "Comparison of Methods to Predict Lower Bound Buckling Loads of Cylinders under Axial Compression," 51st AIAA/ASME/ASCE/AHS/ASC Structures, Structural Dynamics, and Materials Conference, Orlando, FL, 12-15 April 2010, AIAA Paper 2010-2532. 
Table 1. Cylinder geometry and material properties

\begin{tabular}{|c|c|}
\hline Length, $L$ & 31 in. \\
\hline Radius, $R$ & 9 in. \\
\hline Wall thickness, $H$ & $0.040 \mathrm{in}$. \\
\hline Young's modulus & $10.6 \times 10^{6} \mathrm{psi}$ \\
\hline Poisson's Ratio & 0.3 \\
\hline
\end{tabular}

Table 2. Predicted and measured buckling loads

\begin{tabular}{|cc|cc|cc|}
\hline \multirow{2}{*}{} & \multirow{2}{*}{} & \multicolumn{2}{|c|}{ predicted } & \multicolumn{2}{c|}{ measured } \\
\cline { 3 - 6 } & lateral & $\begin{array}{c}\text { local } \\
\text { buckling } \\
\text { test article }\end{array}$ & $\begin{array}{c}\text { global } \\
\text { buckling } \\
\text { load, lb }\end{array}$ & $\begin{array}{c}\text { local } \\
\text { buckling } \\
\text { load, kips }\end{array}$ & $\begin{array}{c}\text { global } \\
\text { buckling } \\
\text { load, kips }\end{array}$ \\
\hline TA01 & 14.7 & 41.7 & 42.0 & $\mathrm{n} / \mathrm{a}$ & 38.0 \\
TA02-local & 24.7 & 27.9 & 40.0 & 23.3 & n/a \\
TA02-global & 24.7 & 27.9 & 40.0 & 24.9 & 37.9 \\
TA06 & 14.7 & 41.7 & 42.0 & n/a & 36.6 \\
\hline
\end{tabular}

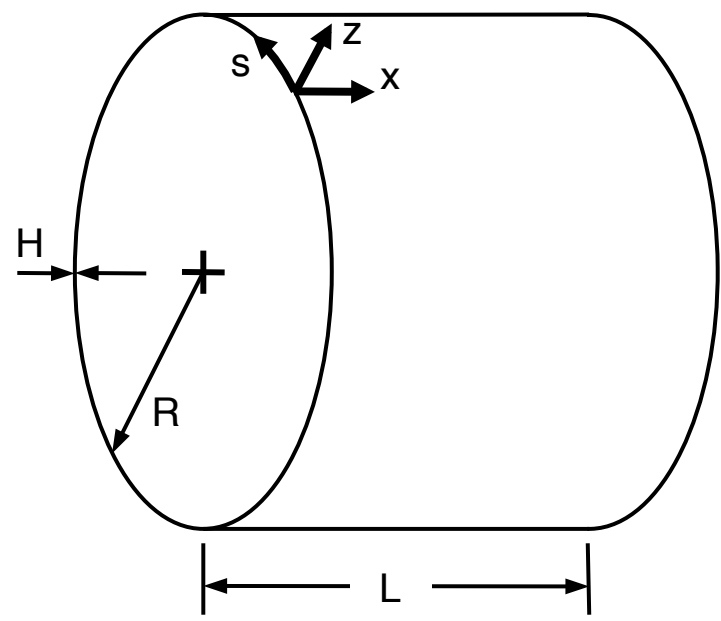

Figure 1. Cylinder geometry and coordinate system 


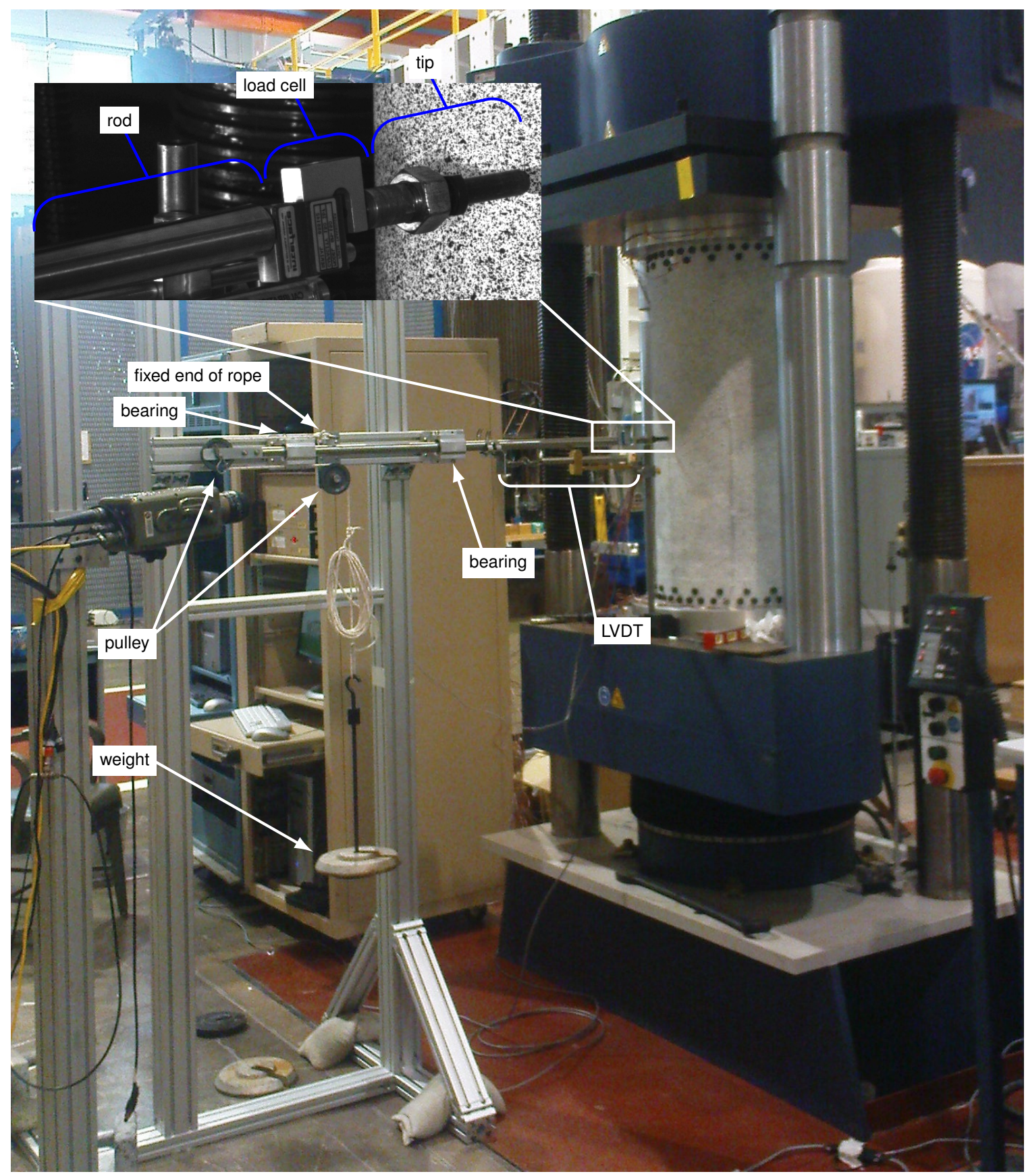

Figure 2. Lateral perturbation apparatus 


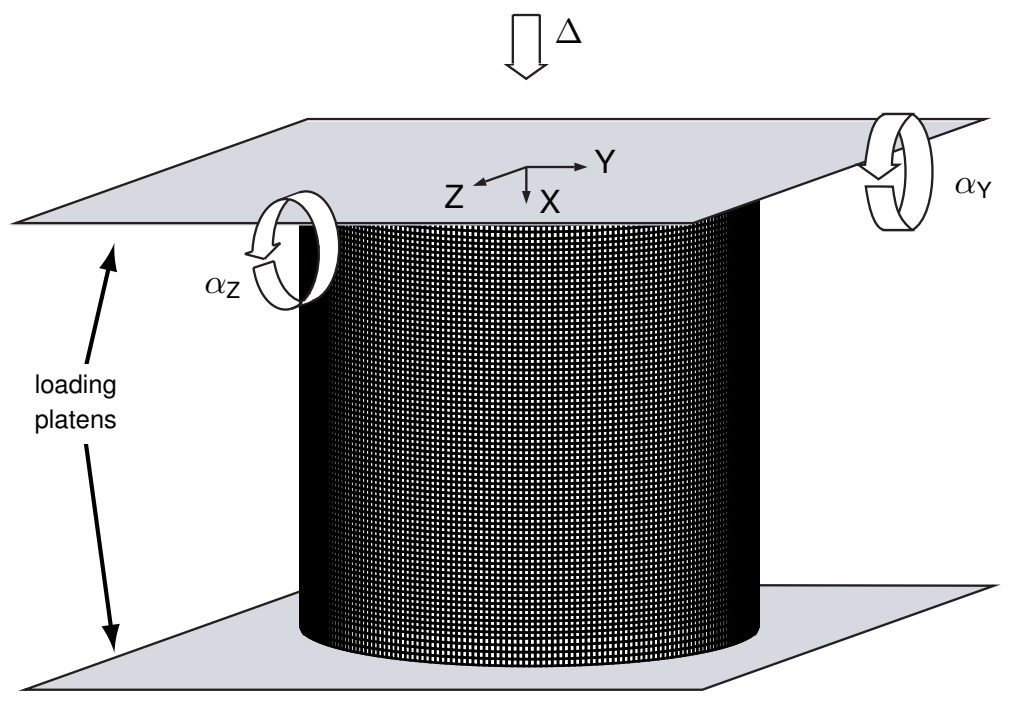

Figure 3. Loading platen rotation angles

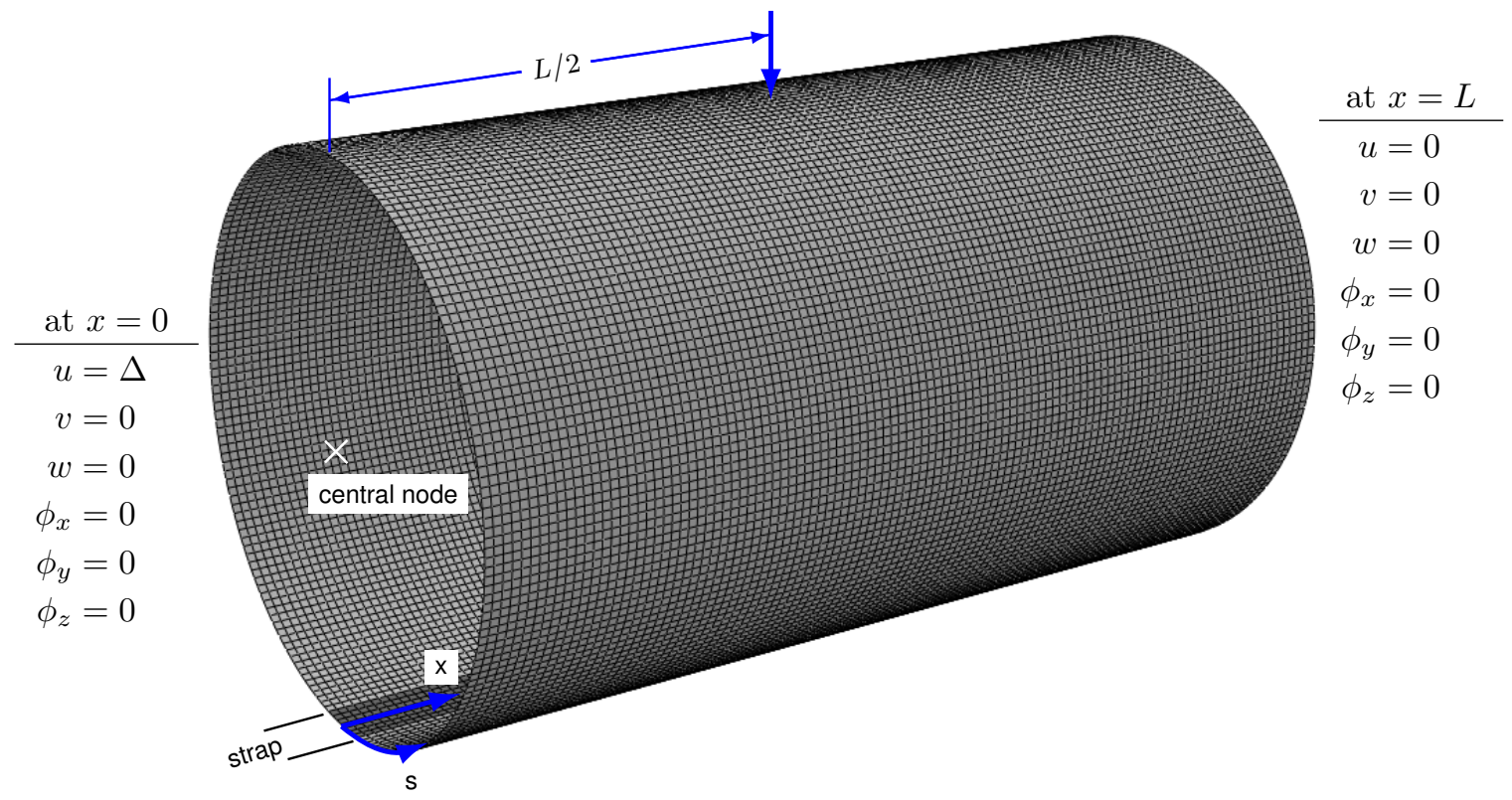

Figure 4. Boundary conditions, loading, and representation of finite-element mesh 


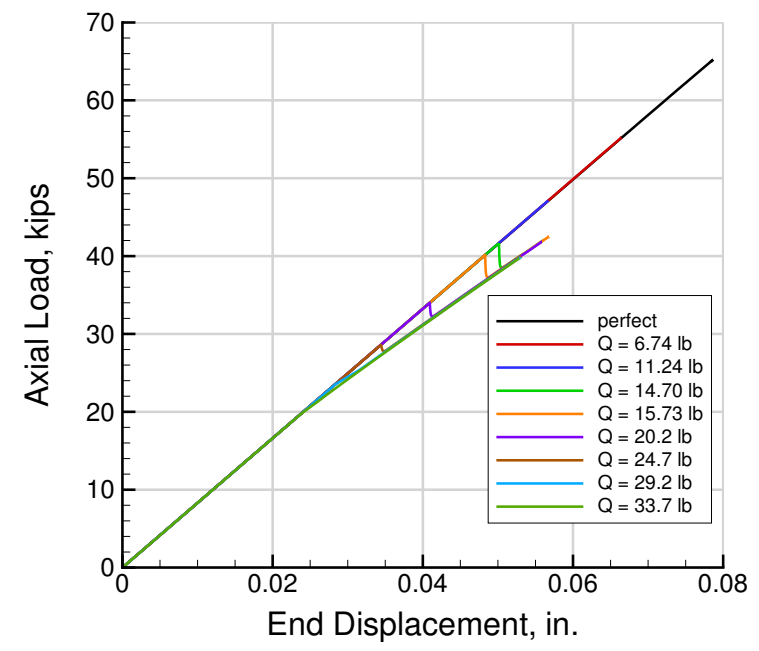

Figure 5. Axial load-end displacement predictions for all lateral load cases

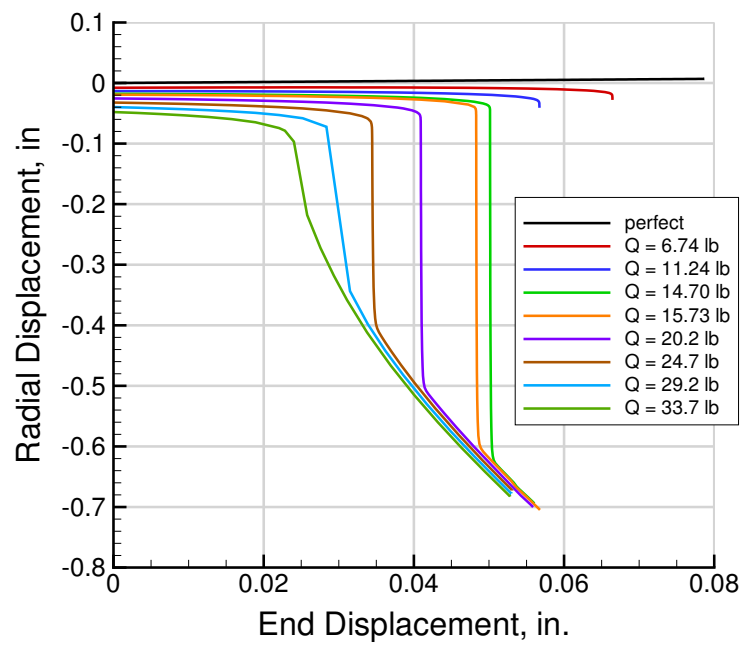

Figure 6. Radial displacement-end displacement predictions for all lateral load cases

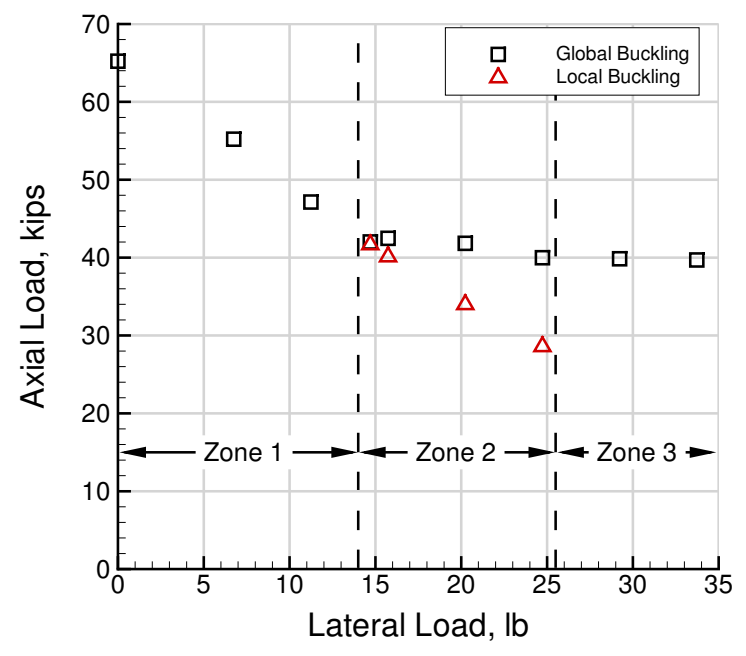

Figure 7. Critical axial loads for all lateral load cases

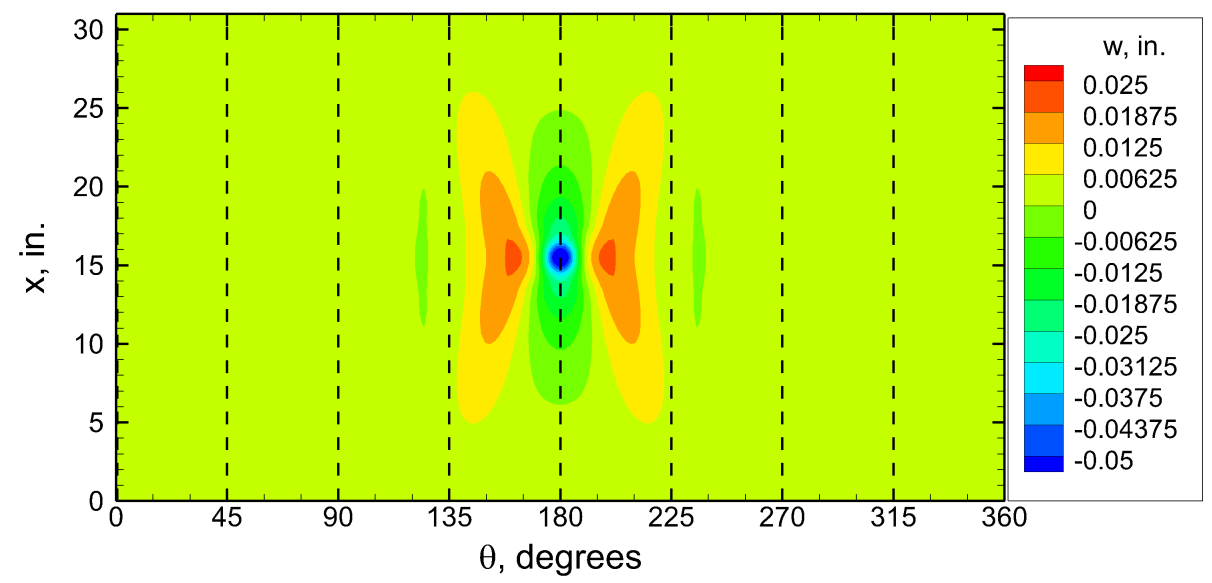

Figure 8. Predicted radial displacements prior to local buckling 


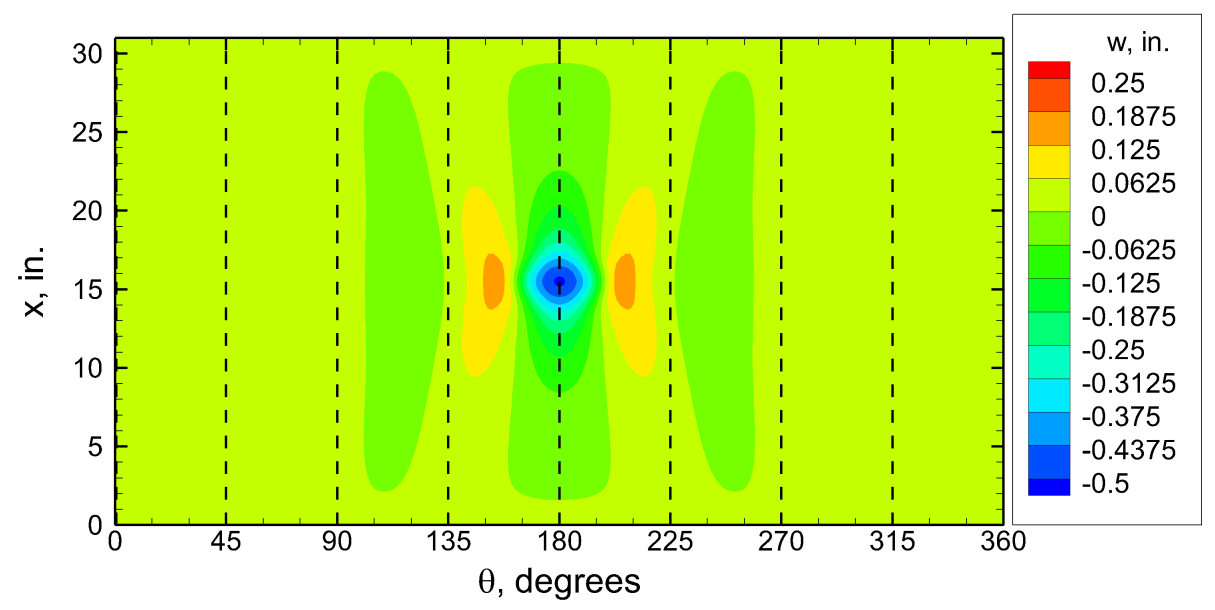

Figure 9. Predicted radial displacements after local buckling

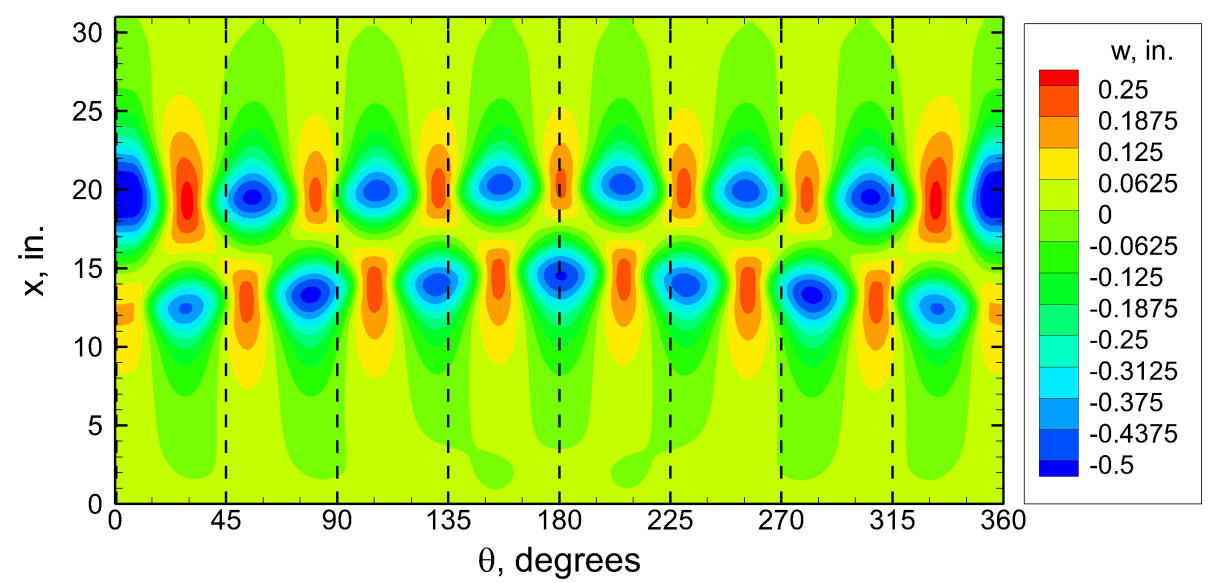

Figure 10. Predicted radial displacements after global buckling

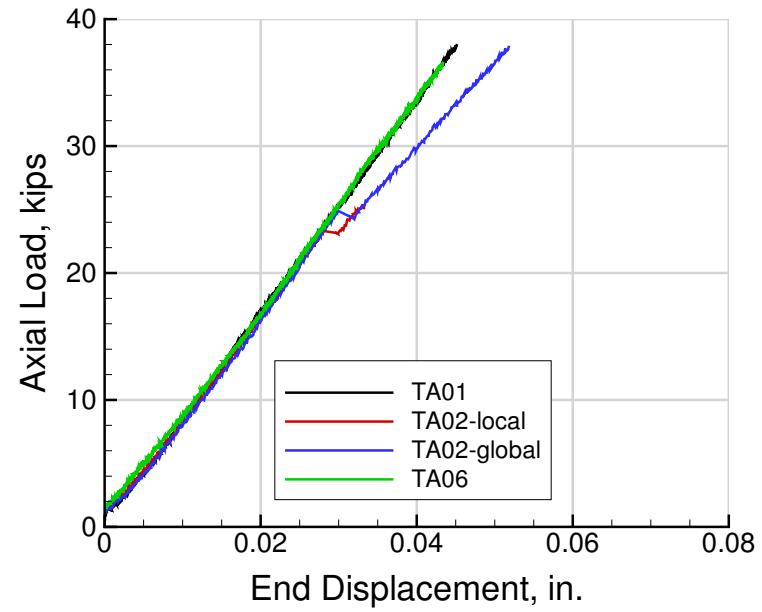

Figure 11. Axial load-end displacement measurements for test cases

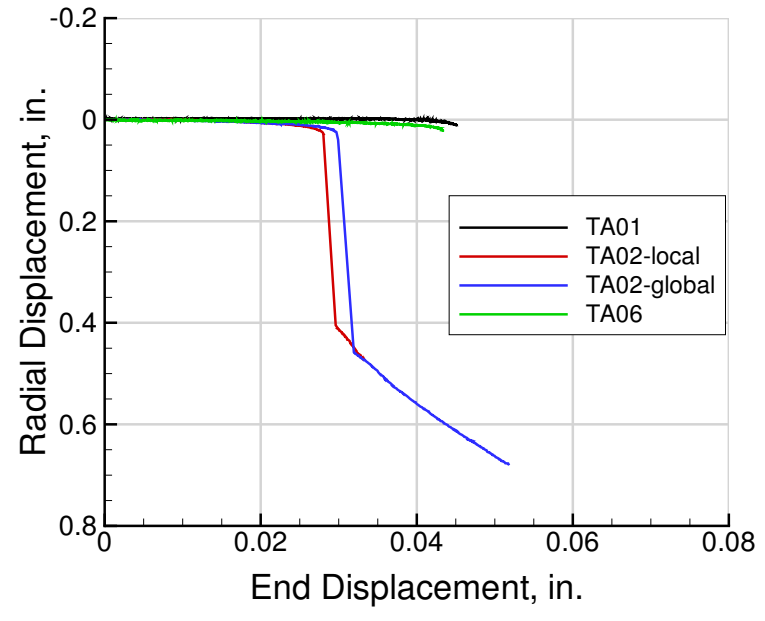

Figure 12. Radial displacement-end displacement measurements for test cases 


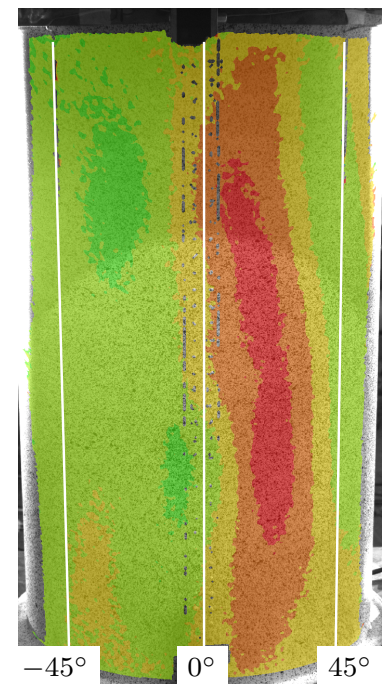

(a) system 3

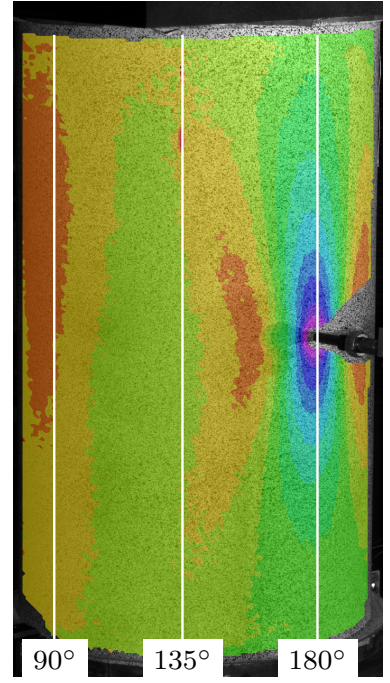

(b) system 1

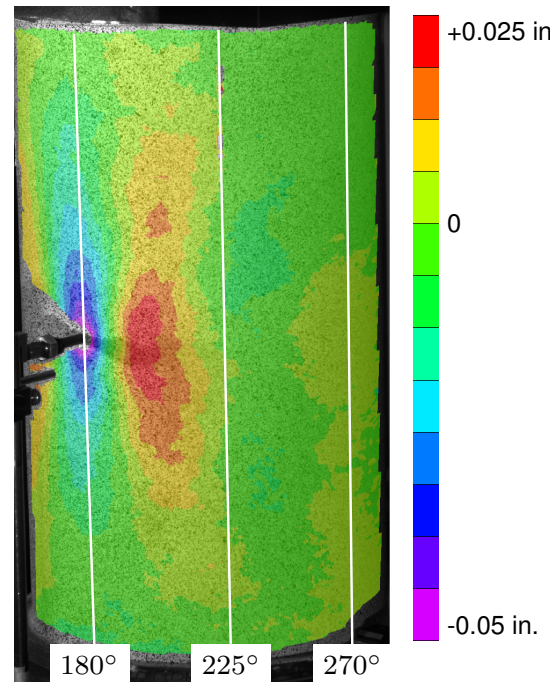

(c) system 2

Figure 13. DIC measurements of radial displacement prior to local buckling in TA02

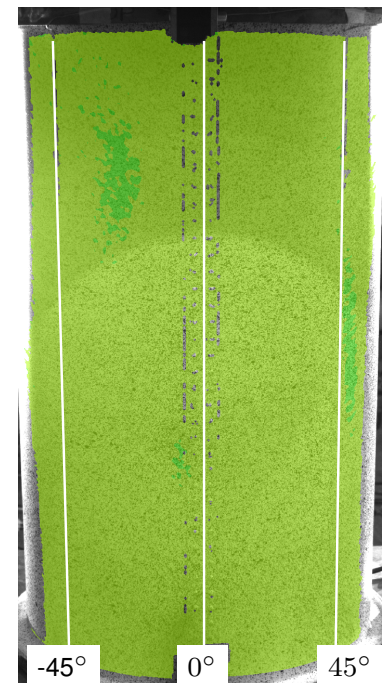

(a) system 3

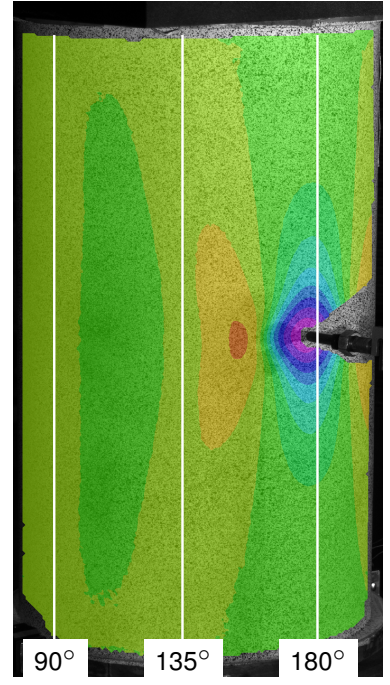

(b) system 1

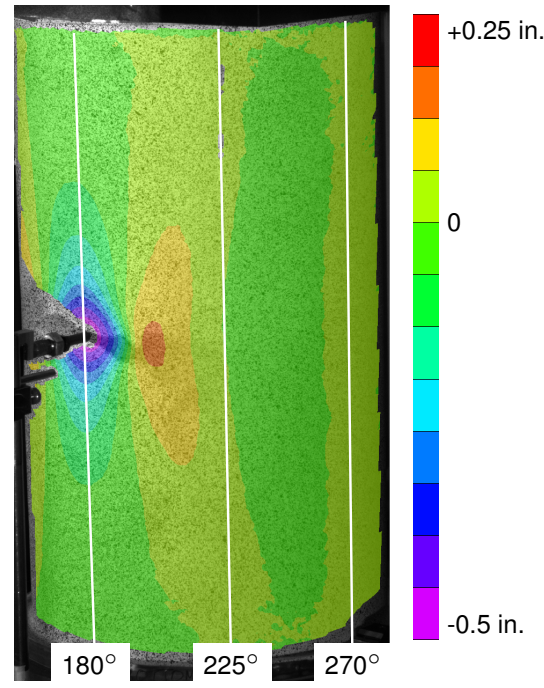

(c) system 2

Figure 14. DIC measurements of radial displacement after local buckling in TA02 


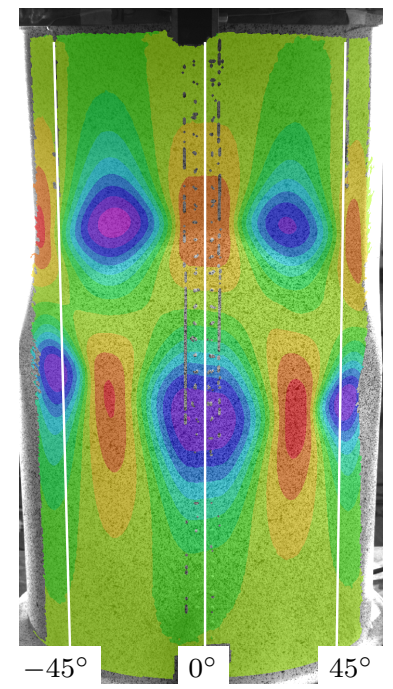

(a) system 3

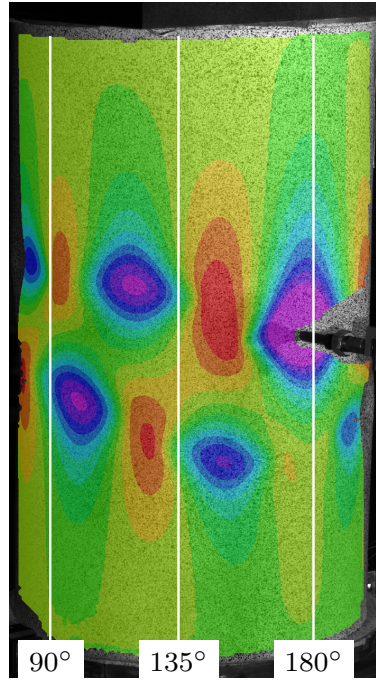

(b) system 1

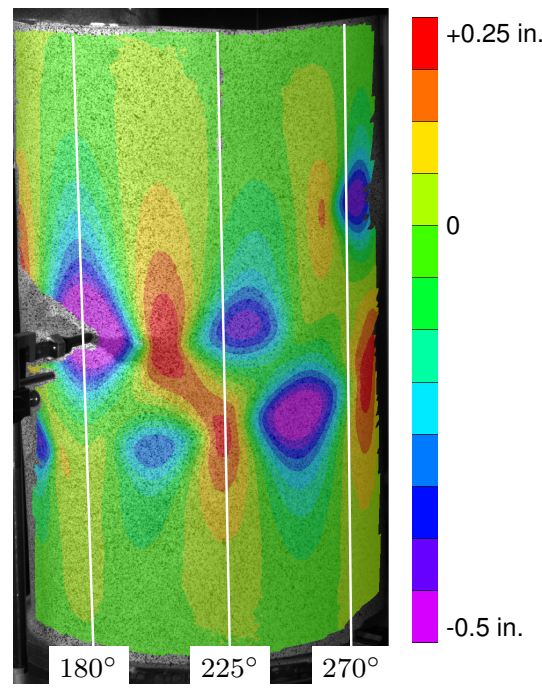

(c) system 2

Figure 15. DIC measurements of radial displacement after global buckling in TA02

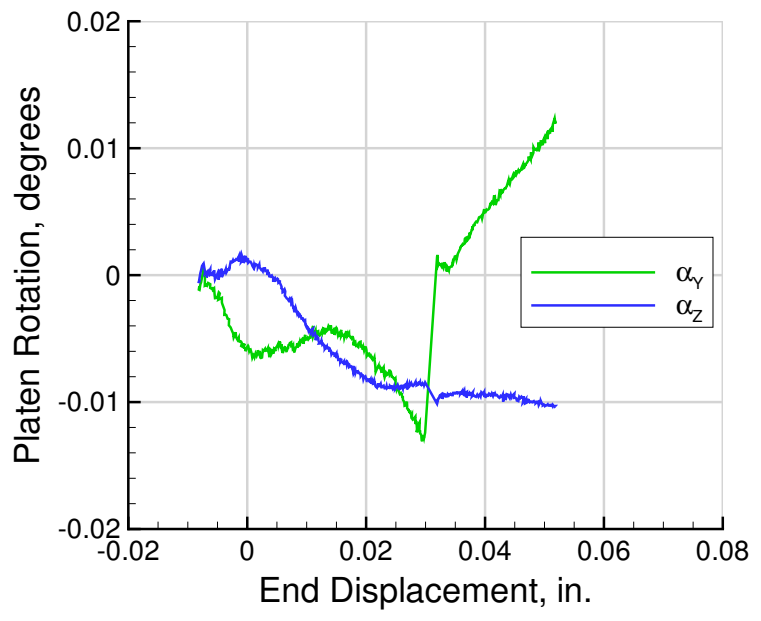

Figure 16. Axial load-end displacement measurements for test cases

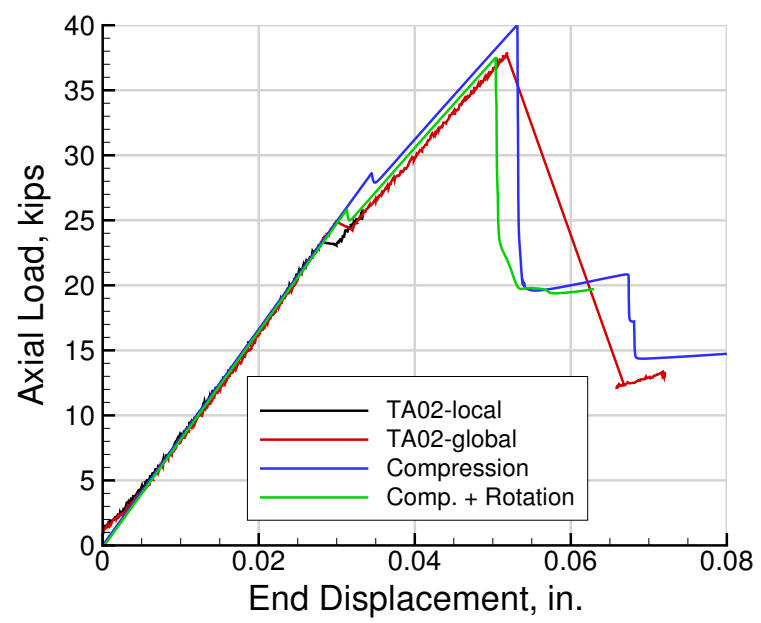

Figure 17. Radial displacement-end displacement measurements for test cases 